\title{
Reflets
}

Revue ontaroise d'intervention sociale et communautaire

\section{Triple journée de travail des mères d'enfants ayant une incapacité invisible}

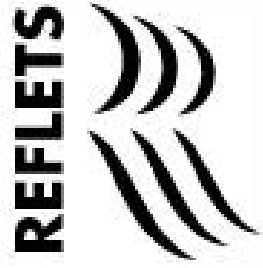

\section{Alice Home et Stéphanie Pearce}

Volume 9, numéro 2, automne 2003

Travail et mieux-être

URI : https://id.erudit.org/iderudit/011095ar

DOI : https://doi.org/10.7202/011095ar

Aller au sommaire du numéro

Éditeur(s)

Reflets : Revue ontaroise d'intervention sociale et communautaire

ISSN

1203-4576 (imprimé)

1712-8498 (numérique)

Découvrir la revue

Citer cet article

Home, A. \& Pearce, S. (2003). Triple journée de travail des mères d'enfants ayant une incapacité invisible. Reflets, 9(2), 163-185.

https://doi.org/10.7202/011095ar

\section{Résumé de l'article}

Les mères d'enfants ayant des incapacités sont plus présentes qu'autrefois sur le marché du travail. Toutefois, leurs expériences sont méconnues. Cet article présente des résultats d'entrevues effectuées auprès de onze mères franco-ontariennes qui cumulent un emploi et les soins à dispenser à un enfant ayant le trouble d'hyperactivité avec déficit de l'attention (THADA). Ces mères vivent plusieurs défis reliés aux comportements de l'enfant et aux attitudes négatives. Ces deux défis ont beaucoup d'impact sur leur vie familiale, personnelle et professionnelle. Compte tenu du fardeau et du peu de soutien disponible, elles doivent accomplir une triple journée de travail ou faire des concessions sur le plan de leur carrière.
Tous droits réservés (C) Reflets : Revue ontaroise d'intervention sociale et communautaire, 2002
Ce document est protégé par la loi sur le droit d'auteur. L'utilisation des services d’Érudit (y compris la reproduction) est assujettie à sa politique d'utilisation que vous pouvez consulter en ligne.

https://apropos.erudit.org/fr/usagers/politique-dutilisation/ 


\title{
Triple journée de travail des mères d'enfants ayant une incapacité invisible
}

\author{
Alice Home \\ Professeure, École de service social, Université d'Ottawa
}

Stéphanie Pearce

Travailleuse sociale, Conseil des écoles catholiques de langue française, Ottawa $^{1}$

\section{Introduction}

On sait très bien que, dans la société, le rôle de prodiguer des soins aux enfants est attribué en premier lieu aux femmes et ce, en dépit de leur entrée massive sur le marché du travail (Institut Vanier 1998). Les aménagements économiques, tant dans le monde du travail que dans la communauté, se basent toujours sur la prémisse de leur entière disponibilité à ce rôle (Cancian et Oliker 1998). En raison d'un certain recul dans la responsabilité publique à l'égard des personnes vulnérables et dépendantes, la demande pour le travail féminin non rémunéré s'est récemment accrue (Gerson 1998). Pour les femmes, ce recul correspond a un accroissement de l'intensité et de la complexité du fardeau que représentent les soins et les besoins familiaux: leur identification, leur planification, les suivis, la recherche de ressources adéquates et la revendication lorsque ces besoins ne sont pas comblés (Lee 1998; Devault 1999). De la part des gouvernements, le transfert de la responsabilité des soins vers la communauté et, par 
conséquent, vers les familles et les femmes, a réduit leur éventail des choix professionnels auparavant acquis, augmentant d'autant leur vulnérabilité économique (Bullock 1990). D'ailleurs, l'emploi de termes tels «aidantes naturelles» ou «amour maternel» ne fait que masquer les désavantages qui affectent les femmes.

La présence des femmes sur le marché du travail est, entre autres, liée à des raisons économiques. Par ailleurs, le fait d'occuper des rôles multiples s'avère bénéfique pour leur santé mentale et ce, en dépit du stress supplémentaire que cela peut engendrer (Home 1997). Pourtant, seule une minorité de mères d'enfants ayant une incapacité occupait, jusqu'à récemment, un emploi (Marcenko et Meyers, 1991; Mardiros 1985). Compte tenu de la lourdeur des responsabilités familiales, la nécessité d'y répondre pendant la journée et l'insuffisance de services de garde adaptés, plusieurs de ces mères ont cédé à la pression sociale de l'assignation domestique et sont retournées à leur rôle traditionnel (Traustadottir 1991; Willoughby et Glidden 1995). Des études récentes indiquent que celles qui occupent encore un emploi à temps plein, le font au détriment de leur temps personnel, cumulant ainsi une triple journée de travail (Jenkins 1997). D’autres sacrifient leur plan de carrière, en réduisant leurs heures au travail ou en ne travaillant qu'à temps partiel, malgré le peu de sécurité ou de bénéfices qu'offre ce type d'emploi (Rocher Institute 2000; Shearn et Todd 2000).

Les rares recherches sur le sujet indiquent que le soutien informel et formel s'avèrent d'une grande importance pour les femmes qui tentent de gérer cette situation fort exigeante de rôles multiples. Cependant, l'absence de recherche ne facilite pas la tâche des intervenantes et intervenants qui voudraient identifier les besoins de ces mères et savoir comment mieux les soutenir. En français, les études et ouvrages pertinents sont quasi inexistants (Tétreault et Blanchette 1991). Cet article vise à combler en partie ces lacunes, en présentant certains résultats d'une étude qualitative effectuée auprès de mères franco-ontariennes qui cumulent un emploi et les soins dispensés à un enfant ayant une incapacité invisible, en l'occurrence, le trouble d'hyperactivité avec déficit de l'attention (THADA). Cette incapacité neurologique touche 
environ $5 \%$ de la population, en plus de constituer la raison principale de consultation des services de santé mentale pour enfants (Barkley 1997).

Plusieurs recherches en psychologie ou psychiatrie abordent ce trouble, mais peu d'études cherchent à connaitre l'expérience des mères (Viola 1997). Entreprise en collaboration avec un groupe d'entraide, notre recherche voulait donner à ces mères l'occasion de s'exprimer sur leur vécu, d'identifier le soutien disponible ainsi que les besoins non comblés. Après avoir résumé la documentation pertinente, nous présentons la méthodologie utilisée, le profil des participantes et les résultats qui touchent les perceptions que les mères se font des ressources disponibles et de celles dont elles auraient besoin. En guise de conclusion, nous discutons des conséquences découlant des résultats obtenus sur les politiques et les pratiques s'adressant à cette population.

\section{Recension des écrits}

Nous présentons, dans cette section, les écrits traitant de la qualité du rôle parental ainsi que ceux concernant l'impact, sur la famille et sur la mère, d'un enfant ayant une incapacité. Le concept de la «qualité du rôle» fait référence au degré d'équilibre entre la gratification et les défis attachés aux rôles (Baruch et Barnett 1986). Les recherches indiquent que la qualité de l'expérience est plus importante que le nombre de rôles assumés. À cet égard, le rôle de mère occupe une signification particulière. En effet, les mères qui exercent un rôle parental de bonne qualité jouissent d'une meilleure santé mentale que celles pour qui les défis excèdent les récompenses (Barnett et al.1994). Cependant, aucune étude n'aborde la qualité du rôle parental des mères dont l'enfant a une incapacité, malgré le fardeau associé aux exigences persistantes et imprévisibles et à la restriction qu'elles subissent sur le plan des options d'emploi (Shearn et Todd 2000; Seligman et Darling 1997). 
"Quand plusieurs conditions difficiles de vie s'ajoutent à une incapacité invisible comme le THADA, ce qui est souvent le cas chez les familles qui consultent en service social, les risques de problèmes familiaux et d'atteinte à la santé mentale augmentent. "
Plusieurs études examinent l'impact d'une incapacité chez l'enfant sur la famille, sans distinguer l'expérience des mères et celle des pères. Le nombre et l'âge des enfants, la composition de la cellule familiale (biparentale ou monoparentale), de même que le revenu, peuvent affecter l'expérience parentale (Sloper 1999). Quand plusieurs conditions difficiles de vie s'ajoutent à une incapacité invisible comme le THADA, ce qui est souvent le cas chez les familles qui consultent en service social, les risques de problèmes familiaux et d'atteinte à la santé mentale augmentent (Biederman et al.1995). La sévérité de l'incapacité peut augmenter le stress en plus de peser lourdement sur la décision de la mère d'occuper ou non un emploi (MacDonald, Poertner et Pierpont 1998). L'absence de garderies adaptées ou de politiques familiales souples en milieu de travail peut aussi avoir une influence sur cette décision (Jenkins 1997). Cependant, plus que la sévérité, des problèmes de comportement, comme l'agressivité ou la provocation peuvent avoir un impact sur le stress parental (Floyd et Gallagher 1997). Lorsque ces problèmes, souvent imprévisibles, relèvent d'une incapacité invisible, le public, ne reconnaissant pas un handicap réel chez l'enfant, tend à le rejeter (Fewell 1990). C'est particulièrement le cas des enfants atteints du THADA. Ce trouble d'origine surtout génétique, entraîne des difficultés importantes au plan des fonctions cognitives d'organisation et de planification, et de la maîtrise du comportement, donc, à un degré inhabituel à l'âge de l'individu et des agissements inhabituels (Barkley 1997).

Les personnes qui en sont atteintes peuvent avoir des problèmes de concentration ou d'organisation, des tendances à agir avant de penser, un retard de développement sur le plan socio-affectif ainsi que de la difficulté à réagir conformément aux attentes de l'entourage (Jacobs 1998; Barkley 1995; Gagnon 2001). Dans plus de la moitié des cas, cette incapacité s'accompagne d'un autre trouble psychiatrique ou d'apprentissage dont les symptômes peuvent s'ajouter aux difficultés vécues par l'enfant et sa famille (Viola 1997; Snyder 2000). En effet, si le THADA ressemble, à certains égards, à d'autres incapacités invisibles comme l'épilepsie, il s'en distingue par le manque de compréhension publique face 
"Les jeunes ayant cette incapacité sont souvent rejetés par les pairs et critiqués par le personnel scolaire à cause de leur comportement dérangeant. En même temps, leurs mères sont blâmées pour les avoir «mal élevés»." à la nature non intentionnelle et involontaire des symptômes. On pense que, si ces jeunes d'apparence «normale» sont peu capables de se contrôler, c'est de leur faute ou de celle de leurs parents (Barkley 1997). Les jeunes ayant cette incapacité sont souvent rejetés par les pairs et critiqués par le personnel scolaire à cause de leur comportement dérangeant. En même temps, leurs mères sont blâmées pour les avoir «mal élevés». Les médias ont d'ailleurs aggravé ce problème, en insistant sur l'existence d'une controverse sur la nature du THADA. Pourtant, les études scientifiques s'accordent pour affirmer que ce trouble est une incapacité biologique réelle (Barkley 1997). Compte tenu de cette incompréhension sociale qui s'ajoute aux problèmes de gestion de l'incapacité, on ne sera pas surpris de l'impact énorme d'un enfant atteint du THADA sur la famille, particulièrement sur la mère sur qui repose le fardeau des soins. En effet, ces femmes sont isolées, plus stressées et plus déprimées que d'autres mères (Baker et McCal 1995), surtout, quand s'ajoutent des contraintes financières (Baldwin, Brown et Milan 1995). Si le soutien informel des proches est absent et peu de ressources formelles disponibles, ces mères risquent de travailler sans arrêt parce qu'elles doivent répondre aux demandes de la sphère du travail rémunéré et à celles de la sphère domestique.

\section{Méthodologie de l'étude}

Nous avons entrepris une étude qualitative qui demandait directement aux mères d'enfants atteints du THADA comment elles vivaient leur expérience et la conciliait avec un emploi. Faisant partie d'une recherche subventionnée ${ }^{1}$, cette étude comporte également un sondage au niveau national. Les deux volets se concentrent sur les mères ayant un emploi d'au moins dix heures par semaine, en plus de s'occuper d'un enfant de six à 17 ans, chez qui on a diagnostiqué le THADA. Nous avons ciblé les enfants d'âge scolaire, parce que l'école peut être à la fois une source de soutien et de stress. L'étude qualitative s'est déroulée à l'aide 
d'entrevues semi dirigées, afin de favoriser l'expression des sentiments et des perceptions tout en s'assurant que les propos restent centrés sur les thèmes particuliers. Cette combinaison de souplesse et de structure s'avère particulièrement adaptée à cette population, certaines mères pouvant elles-mêmes présenter des difficultés d'attention reliées au THADA. Le guide d'entrevue, testé dans les deux langues, traitait des situations de travail et de vie familiale, des difficultés et des gratifications associées au rôle de mère dans cette situation précise, des soutiens reçus et de ceux requis, ainsi que de l'expérience de conciliation d'un emploi avec la responsabilité d'un enfant ayant cette incapacité.

L'échantillon comprenait des mères anglophones et francophones de la région d'Ottawa. Nous les avons jointes grâce à deux moyens. La stratégie principale par contraste (Patton 1994) visait à recruter des mères dans des situations familiales et d'emploi diversifiées. Deux groupes d'entraide ont vu à la promotion de l'étude et plusieurs agences sociales ont diffusé l'information à leur personnel en plus de rendre disponibles dans leur salle d'attente des dépliants bilingues. La collaboration de ces agences s'est avérée indispensable, particulièrement dans le recrutement des participantes franco-ontariennes. Les dépliants, disponibles également dans certains endroits publics, expliquaient les buts et procédures de l'étude, ainsi que les aspects éthiques, en plus d'indiquer comment prendre rendez-vous avec l'équipe. Cette stratégie a permis de recruter environ $70 \%$ des participantes. Elle a été accompagnée de la procédure «boule de neige» (Patton 1994), laquelle consistait à demander aux participantes de distribuer de l'information et un dépliant à d'autres mères aux prises avec le même problème.

D'avril à septembre 2002, nous avons rencontré 40 mères, dont 11 Franco-Ontariennes. Les deux chercheures, elles-mêmes mères d'enfants atteints du THADA, ont dû faire preuve de beaucoup de flexibilité afin de s'adapter à l'horaire lourd et à la situation familiale compliquée des mères interviewées (Home et Pearce 2002). Ces dernières ont annulé des rendez-vous à cause de crises familiales ou d'oubli parce qu'elles étaient elles-mêmes atteintes du THADA. Même s'il fallait parfois remettre le rendez- 
vous, ces mères montraient beaucoup de détermination à se faisant entendre. Certaines francophones se disaient prêtes à s'engager dans des efforts éventuels pour mettre sur pied un réseau ou un groupe d'entraide à l'intention des mères d'expression française. Après l'entrevue, nous avons remis aux participantes une trousse de ressources, en plus de discuter de toutes leurs questions. Les entrevues, enregistrées et transcrites à l'aide du logiciel Ethnograph, ont été codifiées en utilisant une procédure mixte (l'Écuyer 1985), selon laquelle une liste préliminaire de catégories est établie après lecture attentive de quelques entrevues. Une fois atteint un accord inter chercheures sur les catégories (Huberman et Miles 1991), trois chercheures ont codifié les autres entrevues, en s'assurant de se consulter sur tout segment ambigu. À partir des données regroupées par catégories, nous avons élaboré des tableaux synthèses, en vue d'identifier les tendances principales.

Compte tenu de la problématique et des objectifs fixés, la méthodologie employée était efficace et pertinente. Cependant, les procédures empruntées comportent quelques limites, autres que celles reliées au nombre de personnes jointes. La stratégie d'échantillonnage par contraste a permis de recruter des femmes vivant diverses situations familiales. Cependant, la plupart travaillaient dans la fonction publique. Les mères employées dans le secteur privé ou dans certains types de travail plus précaire n'avaient probablement pas le temps de participer à une entrevue. De plus, à cause de certaines difficultés de recrutement (par exemple, des hôpitaux et quelques conseils scolaires exigeaient des procédures éthiques internes), des sous populations de mères n'ont peut être pas entendu parler de l'étude.

\section{Résultats : profil des participantes}

Les onze francophones partageaient plusieurs caractéristiques avec les autres participantes, tout particulièrement sur les plans de leur situation familiale et du type d'emploi. Deux tiers des participantes franco-ontariennes vivaient en couple et la majorité de leurs 
«...plus de $70 \%$ des mères francophones vivaient avec plus d'une personne atteinte du THADA ou d'un autre trouble, comparé au quart dans l'échantillon global. De plus, presque $75 \%$ des enfants francophones avaient un diagnostic additionnel...» enfants ayant le THADA avaient entre six et douze ans. Elles travaillaient toutes à temps plein, la plupart dans les domaines de la santé ou de l'éducation. Cependant, deux d'entre elles avaient pris un congé de maladie récemment, justement à cause des difficultés à concilier leur emploi avec de lourdes exigences familiales. Si d'autres études ont démontré que certaines mères d'enfants ayant des incapacités étaient obligées d'interrompre leur emploi (Traustadottir 1991), il faut souligner que les participantes en question s'occupaient chacune de deux enfants avec le THADA et dans un cas, un enfant cumulait aussi un trouble d'apprentissage. D'ailleurs, plus de $70 \%$ des mères francophones vivaient avec plus d'une personne atteinte du THADA ou d'un autre trouble, comparé au quart dans l'échantillon global. De plus, presque $75 \%$ des enfants francophones avaient un diagnostic additionnel, soit un trouble d'apprentissage, d'humeur, d'anxiété, un trouble oppositionnel ou un syndrome (Tourette ou Asperger). Ce taux est beaucoup plus élevé que celui de $50 \%$ observé dans l'échantillon global et rapporté dans d'autres études (Snyder 2000). Il semble que les participantes franco-ontariennes étaient confrontées à des exigences familiales particulièrement lourdes.

\section{Vivre avec un enfant qui a une incapacité: résultats concernant la qualité du rôle maternel}

"Certaines mères ont été incapables d'identifier des gratifications, mises à part certaines bonnes journées, sans trop de disputes et où l'enfant avait un visage souriant. "
Un aspect unique de cette étude est que nous avons cherché à connaître, non seulement les défis, mais aussi les gratifications rencontrées, en tant que mère d'un enfant atteint du THADA. Certaines mères ont été incapables d'identifier des gratifications, mises à part certaines bonnes journées, sans trop de disputes et où l'enfant avait un visage souriant. D'autres mères ont indiqué trois types de gratifications. Celle qui revient le plus souvent est la présence de qualités intéressantes chez l'enfant, telles la créativité, l'intelligence, certains talents, leur nature aventureuse, leur perspicacité, leur énergie débordante ou leur habileté à amuser 
les autres. À titre d'exemple, une mère indique: «Il a tellement d'énergie et il est tellement comique, il nous fait rire pendant des heures». Un autre type de gratification vient du progrès de l'enfant, en dépit de son incapacité. Comme l'a dit une participante: «Quand quelque chose clique, quand il réussit bien à l'école [...] on sait qu'il met deux fois plus d'efforts que d'autres enfants dans la classe pour accomplir le même travail». Enfin, pour quelques mères, le fait d'aider leur enfant à affronter les difficultés leur a ouvert de nouvelles perspectives. Grâce à leur expérience maternelle fort exigeante, ces femmes ont vécu une croissance au plan personnel ou ont acquis de nouvelles habiletés.

De nombreux défis sont ressortis. La plupart des mères voient leur rôle maternel comme étant toujours difficile, même s'il y a des journées ou des années plus faciles. L'équilibre perçu entre les difficultés et les gratifications semble varier selon, entre autres, l'âge de l'enfant ou sa situation scolaire. Il y a des journées où elles voudraient tout abandonner, particulièrement lorsqu'il y a des plaintes fréquentes venant de l'école. Il y a des âges plus difficiles, par exemple, lorsque les enfants ont entre 12 et 14 ans et que l'«on sait qu'à cet âge-là, ils ne devraient pas être comme ça». Ce résultat reflète probablement le retard de développement d'environ 30 \% dans la capacité de contrôler ses comportements (Barkley 1997). Plusieurs mères disent vivre des moments plus positifs, quand elles ont une bonne communication avec l'enfant ou le personnel scolaire. Par contre, quelques mères, dont les enfants sont très contrariants, trouvent entièrement négatif leur rôle maternel: "C'est toujours une bataille, neuf fois sur dix».

Certaines des difficultés identifiées relèvent directement des incapacités de l'enfant. Lorsqu'il s'agit de problèmes cognitifs d'attention et d'organisation, les mères doivent tout surveiller et répéter les directives. L'une d'entres elles décrit sa routine matinale: «Je dois lui dire une étape à la fois ce qu'il doit faire. Mets ton bas. Mets ton autre bas. Mets ton pantalon... Ceci ne se fait pas aussi facilement que ça car lorsqu'il a mis un bas, il tombe dans les nuages et perd son temps au lieu de finir de s'habiller». Durant la période des devoirs, lorsque l'enfant n'écoute pas les directives ou qu'il est incapable de se concentrer, «un devoir qui devrait 
"C'est souvent la mère qui doit tout laisser tomber pour essayer de réparer les pots cassés, entre autres avec l'école où se vivent des crises fréquentes de la part de ces enfants. Arrivant surtout durant la journée, ces situations impliquent du côté de la mère des négociations difficiles avec son milieu de travail." prendre quinze minutes prend une heure et demie», particulièrement si l'enfant présente en plus un trouble d'apprentissage. Il n'est évidemment pas question de matins productifs ou sereins, ni de soirs qui permettent un certain repos après la journée de travail.

Lorsque l'hyperactivité et l'impulsivité sont présentes, cela mène à des problèmes sociaux et des comportements qui dérangent l'entourage, causent le rejet de la part de leurs camarades et entraînent des suspensions de l'école ou l'expulsion des activités sportives et récréatives. À cet égard, une participante indique: «Ils réagissent vite. Des fois les coups de poings vont revoler avant qu'ils aient pensé». Une autre affirme: «S'il a envie de dire quelque chose, il pensera pas comment le dire pour pas blesser». C'est souvent la mère qui doit tout laisser tomber pour essayer de réparer les pots cassés, entre autres avec l'école où se vivent des crises fréquentes de la part de ces enfants. Arrivant surtout durant la journée, ces situations impliquent du côté de la mère des négociations difficiles avec son milieu de travail. Cela peut influencer le type d'emploi qu'elle peut donc exercer ainsi que sur ses chances d'avancement.

Si les médicaments peuvent aider à gérer le THADA chez la plupart des enfants, ce traitement n'est pas sans inconvénient, à cause des effets secondaires, des problèmes de dosage ou de résistance d'un enfant plus âgé à la prise du médicament. Toutefois, c'est la réaction de l'entourage au fait qu'on a recours à ce type de traitement qui dérange le plus les mères, comme l'a affirmé une participante: «C'est difficile le fait qu'ils prennent des médicaments. Des fois on se sent juger». En effet, la deuxième catégorie de difficultés rapportées par les mères dépasse la gestion des symptômes, pour englober des défis qui affectent plus globalement la famille et les mères. Les mythes et préjugés par rapport aux médicaments ne sont qu'un exemple des nombreuses attitudes négatives que rencontrent les mères et qui rendent encore plus difficile un rôle déjà exigeant. Plusieurs participantes ont déploré l'incompréhension, le préjugé ou le rejet, qui reflètent un manque d'information et de tolérance par rapport à cette 
incapacité en particulier. Une mère explique que "L'entourage ne comprend pas toujours ce qu'on peut vivre comme famille». Une autre dit que «Les gens ont tendance à juger quelque chose qu'ils ne comprennent même pas. Je me sens jugée dans mon rôle de mère». Ainsi, non seulement les enfants sont-ils rejetés, mais la famille entière est exclue des activités et des réseaux, parce qu'on ne veut pas s'associer ni aux enfants, ni aux mères qu'on tient responsables des comportements socialement inacceptables. Ces dernières sont alors obligées d'instruire la parenté, la communauté et même, certains professionnels en milieu scolaire et médical. À défaut d'une sensibilisation de la part des mères, ces personnes risquent de s'attendre à des améliorations instantanées du comportement ou de la performance scolaire, sans tenir compte de la nature chronique de cette incapacité. D'ailleurs, plusieurs participantes nous ont confié leur déception face à l'irréalisme de telles attentes.

La plupart des personnes minimisent l'impact d'un enfant atteint du THADA sur la famille. Celle-ci est astreinte à un horaire rigide et elle doit assurer une discipline constante. Une mère explique: «C'est qu'il faut toujours que tu ne sortes pas de la routine $[. .$.$] il faut toujours qu'il soit dans un carré [. .$.$] le souper$ à telle heure, les devoirs à telle heure. Tu peux pas y dire un petit congé ce soir, un petit film d'une demi-heure». Ces restrictions

"...les tensions de couple peuvent surgir, à cause de l'implication parentale inégale ou des désaccords sur la façon d'éduquer l'enfant atteint $d u$ THADA. Les disputes fréquentes avec l'enfant ou encore entre les enfants peuvent contribuer au climat familial tendu. " ont un impact également sur la vie du couple, pour laquelle il reste peu de temps ou d'énergie. En effet, les tensions de couple peuvent surgir, à cause de l'implication parentale inégale ou des désaccords sur la façon d'éduquer l'enfant atteint du THADA. Les disputes fréquentes avec l'enfant ou encore entre les enfants peuvent contribuer au climat familial tendu.

À cette situation familiale difficile, s'ajoute les contraintes financières reliées aux coûts élevés de certains services professionnels qui ne sont pas couverts par l'assurance-maladie. Les mères peuvent alors se sentir partagées entre l'obligation de travailler à temps plein, pour des raisons financières, et celle d'être disponibles pour gérer les crises associées auTHADA. Quelques mères peuvent absorber les dérangements fréquents au travail grâce à un horaire flexible, mais d'autres sont pénalisées si elles doivent s'absenter 
"Elles hypothèquent aussi leurs heures de sommeil. En raison de la vigilance et de l'implication parentale requise, ces mères trouvent qu'elles n'ont jamais de répit, ni de temps à ellesmêmes." souvent, comme celle qui a manqué deux réunions importantes et «malheureusement, il y a eu des séquelles». Certaines mères décident alors de renoncer au travail à temps plein: «il fallait que j'aille à des rendez-vous chez le psychiatre ou le psychologue, ou fallait que j'aille chercher mon fils à l'école où il était suspendu». D'autres mères essaient de résoudre ce dilemme en changeant d'emploi pour un autre moins stressant, en refusant des promotions ou en retardant des projets d'études postsecondaires. Par contre, aucune participante n'a rapporté que le père avait fait des modifications au niveau de son emploi. Comme l'a dit une des mères: «C'est moi qui fait des concessions».

Certaines femmes sont obligées de travailler à temps plein, particulièrement celles qui sont monoparentales. Cette situation implique alors une triple journée de travail. Une participante compare sa journée à celle d'autres mères qui ont un emploi: «Ton travail finit à quatre heures, pis ton deuxième travail commence après. Mais moi,j'en ai trois, quatre. Ça finit jamais». Ces mères sont obligées de sacrifier leur maigre temps de loisirs, leurs activités ou leurs contacts sociaux. Elles hypothèquent aussi leurs heures de sommeil. En raison de la vigilance et de l'implication parentale requise, ces mères trouvent qu'elles n'ont jamais de répit, ni de temps à elles-mêmes. Lorsqu'elles dorment, elles ne se reposent pas: «Le soir je suis épuisée, je me couche pis je l'entends. Mentalement, il est toujours dans ma tête, je dors pas bien dans le sens que j'ai pas eu mon congé comme d'autres parents en ont». Si elles ne font pas attention, leur santé est affectée et elles finissent par être épuisées, comme les deux participantes qui ont du prendre un congé de maladie. Cependant, elles sont tellement occupées qu'elles ne voient pas venir l'épuisement, comme l'a affirmé l'une des deux: «Tomber comme ça, ça été un gros choc».

Compte tenu des difficultés exprimées par les mères ayant des enfants atteints du THADA, la question suivante se pose. Est-ce qu'on leur offre assez de soutien? 


\section{Soutien informel}

"...l'implication $d u$ père peut être limitée. Lorsqu'il se réfugie dans son emploi, son soutien peut se réduire au plan financier,..."
Il est clair que concilier un emploi avec la responsabilité d'un enfant atteint du THADA comporte des défis considérables. Ce vécu difficile nécessite un soutien accru qui n'est pas toujours disponible. Le soutien offert par les conjoints peut varier d'une famille à l'autre. La plupart des mères interrogées reçoivent un certain soutien du père qui accompagne l'enfant à des rendezvous ou s'implique dans ses activités sportives. Cependant, l'implication du père peut être limitée. Lorsqu'il se réfugie dans son emploi, son soutien peut se réduire au plan financier, laissant la mère «toute seule à élever ma fille !» ou «monoparentale mariée!», comme le déplorent deux participantes. D'autres mères, disent apprécier leurs maris compréhensifs, qui sont reconnaissants de ce qu'elles font et qui leur offrent un soutien constant. Toutefois, elles sont obligées d'insister pour qu' ils s'impliquent dans certaines tâches plus exigeantes (supervision des devoirs, rencontres avec le personnel enseignant). La réussite est difficile: «Faut toute que je l'organise", nous a confié une mère. Un partage très inégal des tâches rend beaucoup plus difficile pour la mère la conciliation entre la vie familiale et la vie professionnelle.

Le soutien du conjoint est primordial quand la parenté est de peu de secours. À l'occasion, la famille étendue, qui accepte l'incapacité de l'enfant et sait s'y prendre avec lui, apporte une aide indispensable. Certains grands-parents gardent l'enfant régulièrement ou le surveillent, en cas de crise à l'école. Quelques sœurs ou belles-sœurs, vivant aussi avec des personnes atteintes du THADA, offrent une écoute et de l'information. Cependant, six des mères interviewées ne reçoivent aucun soutien de la parenté, comme cette participante le constate: «Il y a bien des membres de ma famille qui ne veulent rien savoir de lui $[\ldots]$ ils ont peur de sa réaction». Le soutien affectif, provenant de certains amis ou amies est très apprécié, même si ces derniers font rarement du gardiennage. Cependant, une des mères reçoit du répit d'un couple capable de prendre ses enfants pour quelques fins de 
semaine chaque année. Quelques participantes trouvent du soutien auprès de collègues de travail qui partagent leur vécu de mère ou suggèrent des ressources. Toutefois, lorsqu'elles connaissent d'autres mères d'enfants atteints du THADA, elles se sentent davantage comprises. Dans un cas, les familles "ont cliqué»: "C'est un gros bonding $[\ldots]$ toutes nos activités qu'on fait en famille $[\ldots]$ on les fait avec cette famille-là».

\section{Soutien formel}

Les participantes reçoivent du soutien formel provenant des sources professionnelles, scolaires et communautaires. Le soutien professionnel offert par les médecins de famille, pédiatres, et psychiatres a été souvent mentionné, notamment au chapitre de l'aide apportée pour obtenir un diagnostic ou des médicaments. Plusieurs mères ont apprécié le fait que leur médecin connaisse suffisamment bien le THADA pour procéder à un diagnostic ou pour les référer à un spécialiste pour qu'une évaluation soit faite. Six mères ont souligné la différence que font les médicaments, comme dans ce cas où c'est l'enfant qui constate: «Ma tête, c'est pareil comme une poubelle, y a assez de déchets là-dedans. Depuis que je prends mes médicaments, ma poubelle-là [...] la tornade y passe moins». L'aide apportée dépasse le niveau strictement médical, lorsque des médecins amènent les enfants à s'exprimer sur leur vécu ou suggèrent à la mère des exercices de relaxation et des livres sur le THADA. Dans un cas, le médecin a écrit un rapport à l'intention de l'école, expliquant le problème d'attention de l'enfant, suggérant que celui-ci soit placé en avant de la classe et que les directives pour les projets plus complexes soient consignées par écrit.

D'autres types d'aide professionnelle ont été apportés par des psychologues, des ergothérapeutes et des intervenantes et intervenants sociaux. Si les psychologues sont appréciés pour leur rôle dans l'évaluation, leur soutien a également été signalé au niveau du traitement direct. Un psychologue a suggéré que la 
"Lorsque des services spécialisés sont disponibles, ils sont très appréciés.... Cependant, dans de nombreux cas, une urgence, telle un signalement à la Société d'aide à l'enfance a été à l'origine de l'intervention $d u$ service social. " mère se fasse évaluer pour le THADA, "ce qui a changé ma vie», nous a-t-elle confié, alors qu'un autre a facilité les interactions avec l'école. Des travailleuses et travailleurs sociaux sont allés observer l'enfant à l'école, ont rencontré la famille régulièrement, ont répondu rapidement à une demande de placement temporaire ou sont intervenus pour faire avancer les évaluations ou l'accès aux services. À titre d'exemple, la Société d'aide à l'enfance (SAE) a placé un enfant en famille d'accueil, laquelle s'est alliée avec la mère pour obtenir des services nécessaires.

Lorsque des services spécialisés sont disponibles, ils sont très appréciés. Une mère a souligné l'attitude respectueuse des personnes qui ont évalué et soigné son enfant à l'hôpital. Une autre mère a été soulagée lorsqu'une équipe d'intervention de crise s'est impliquée dans la situation et, quelques participantes ont trouvé utiles les ateliers pour parents d'enfants atteints du THADA. Cependant, dans de nombreux cas, une urgence, telle un signalement à la Société d'aide à l'enfance a été à l'origine de l'intervention du service social. C'est le cas de cet enfant agressif à qui on a fourni une aide au plan de la gestion de la colère. Comme l'a dit une mère dont l'enfant avait des comportements très inquiétants: «C'est [seulement] à partir de là qu'on a commencé à avoir de l'aide».

Les participantes reconnaissent également le soutien obtenu dans le milieu scolaire, comme l'ide de cette «enseignante ressource qui est une perle». Cette dernière a assisté aux réunions avec le directeur d'école, lorsque l'enfant avait des difficultés, en plus de prendre le temps d'écouter la mère et de l'aider à trouver un psychologue. Certaines enseignantes ou enseignants, qui ont reconnu les signes du THADA, ont contribué au dépistage, en suggérant que l'enfant se fasse évaluer ou en le référant aux services appropriés. D'autres membres du personnel scolaire ont collaboré dans le traitement de l'enfant, en administrant les médicaments, en aidant la mère à changer un comportement ou en lui offrant un soutien constant,

Plusieurs mères ont apprécié l'aide reçue d'une source communautaire. Certaines activités récréatives se sont avérées particulièrement appropriées, comme un cours de karaté, qui a aidé 
"...certains services spécialisés ne sont accessibles qu'aux familles qui ont des assurances ou qui ont la capacité d'en défrayer les coûts, alors que les groupes d'entraide sont une source peu coûteuse d'information et de soutien." un garçon à canaliser son agressivité et à mieux se concentrer. Les responsables de ce cours ont également pris l'initiative de donner de l'information sur les Grands Frères. Quelques ressources communautaires sont allées plus loin pour permettre l'intégration des enfants et réduire le risque de rejet. Par exemple, dans un camp de jour, on associe chaque enfant atteint du THADA à un «parrain», étudiant à l'école secondaire, pour le surveiller et l'aider à éviter les problèmes.

En plus de ces services réguliers, il existe des ressources spécialisées, comme des groupes d'habiletés sociales pour les enfants. À titre d'exemple, un groupe d'activités du samedi matin offre aux mères quelques heures de répit. Des ateliers d'habiletés parentales ont permis à quelques mères d'acquérir des outils, tout en les rassurant qu'elles étaient "correctes en tant que parents». Cela dit, certains services spécialisés ne sont accessibles qu'aux familles qui ont des assurances ou qui ont la capacité d'en défrayer les coûts, alors que les groupes d'entraide sont une source peu coûteuse d'information et de soutien.

À cet égard, quelques participantes ont souligné le soutien de l'organisme Children and Adults with Attention Deficit Disorder (CHADD), au niveau des outils et des ressources obtenues et de la prise de conscience que d'autres partagent la même situation. Pour une mère: "C'était ma dose de remontant à tous les mois». Malheureusement, les CHADD ont de la difficulté à maintenir leurs activités, parce que ce sont des bénévoles qui en assument la responsabilité. Comme l'a expliqué une mère: «Il ne faut pas s'attendre à ce que ça soit les parents THADA qui les organisent. On en a assez sur les bras». Par ailleurs, l'équivalent francophone de CHADD ne fonctionne plus depuis quelques années en Ontario.

La pénurie de ressources en français a été soulignée par plusieurs participantes. Par exemple, une mère s'est vue refuser des traitements, parce que l'on ne pouvait pas effectuer l'évaluation en français. Pour accélérer le processus, une autre mère a décidé de faire évaluer son fils en anglais. Ce fut peine perdue; l'enfant éprouvait des difficultés de communication: «Je comprends pas, maman. Qu'est-ce qu'elle dit?». Pour tenter de résoudre le problème des spécialistes unilingues, un couple francophone s'est 
"Insister pour des services en français dans la région d'Ottawa implique une attente qui peut être longue, entre six et dix mois." rendu ensemble au rendez-vous dans l'espoir que l'un ou l'autre comprendrait ce qui se dirait. Insister pour des services en français dans la région d'Ottawa implique une attente qui peut être longue, entre six et dix mois. Quelques mères ayant obtenu des services dans leur langue, attribuent leur succès à leur initiative ou à leur débrouillardise. Mais, la plupart d'entre elles sont d'accord: «les services en français, ça fait dur».

Plusieurs autres besoins non comblés sont ressortis. Les participantes requièrent des évaluations de qualité, abordables et rapides, afin d'éviter que la situation scolaire et familiale ne se détériore. Les mères d'adolescentes et d'adolescents réclament des activités après l'école, les fins de semaine et l'été, compte tenu de leurs besoins d'encadrement et de structure. Or, les ressources disponibles pour les jeunes de ce groupe d'âge s'adressent surtout aux jeunes ayant une incapacité physique ou intellectuelle. Les mères recherchent aussi des groupes qui permettraient à ces adolescentes et adolescents d'acquérir certaines habiletés (sociabilité, gestion de colère), tout en leur offrant une occasion d'échanger avec les pairs vivant la même situation.

Les participantes reconnaissent qu'elles ont elles-mêmes besoin de lieux d'échange et de soutien. Une mère affirme que «juste jaser avec des parents qui vivent des choses semblables c'est la chose qui serait la plus utile». À cet égard, des ateliers d'habiletés parentales, les groupes de soutien avec animation professionnelle et les groupes d'entraide ont été mentionnés. De plus, les participantes ont exprimé un besoin urgent de répit, compte tenu des comportements problématiques de leurs enfants et de la difficulté d'obtenir l'aide requise du côté de la parenté. Comme l'a expliqué une mère: «Une fin de semaine off de temps en temps et de savoir que les enfants sont entre bonnes mains et qu'on ne se fait pas juger parce que l'on prend une fin de semaine pour penser à nous autres». Cependant, les parents d'enfants atteints du THADA n'ont aucun droit au répit, à moins que l'enfant ait d'autres incapacités, comme l'autisme. Cela explique pourquoi certaines participantes ont suggéré aussi qu'il y ait, auprès des gouvernements, de l'action sociale dans le but d'obtenir des subventions pour le gardiennage spécialisé et le répit. 


\section{Discussion et implications}

"Il s'agit clairement d'une triple journée de travail, qui requiert une énergie abondante et une disponibilité constante. "
Les participantes à cette étude présentent un portrait plutôt sombre de leur vécu maternel. Si les difficultés mentionnées ressemblent à celles rapportées dans d'autres recherches portant sur le THADA (Barkley 1995; Bernier et Siegal 1994), notre étude fait ressortir davantage le manque d'équilibre entre les défis et les gratifications, étant donné qu'environ $75 \%$ des participantes vivent des situations familiales très difficiles. L'impact du THADA et des troubles qui l'accompagnent sur les mères rencontrées est similaire, à certains égards, à ce que rapportent des mères dont les enfants ont d'autres types d'incapacités. La quantité énorme de travail non rémunéré et l'attente que la mère en assume le fardeau, peu importe ses autres responsabilités, ne semblent pas varier selon l'incapacité (Tétreault et Blanchette 1991). Il s'agit clairement d'une triple journée de travail, qui requiert une énergie abondante et une disponibilité constante (Cancian et Oliker 1998).

Cependant, les responsabilités de recherche de services, de planification et de suivi sont davantage le lot de la femme, tout particulièrement lorsque le père éprouve aussi des problèmes d'organisation reliés au THADA, compte tenu de l'origine génétique du trouble. Une autre différence relève des problèmes de comportement présents chez la plupart des enfants qui sont atteints du THADA. Non seulement le climat est plus tendu mais, selon l'étude de Bristol et al. (1988), les pères ont tendance à se retirer davantage, comparé aux familles où l'incapacité de l'enfant n'implique pas de tels problèmes. En outre, la société est portée à juger davantage la femme lorsque l'incapacité de l'enfant est invisible, peu comprise et qu'elle se manifeste par les comportements difficiles. Finalement, le fardeau se trouve allégé quand l'entourage reconnait les difficultés de la famille aux prises avec le THADA (Anthony et Fisher 2000) mais nos résultats indiquent que ce soutien n'est pas toujours disponible.

Les problèmes associés au THADA ont également un impact important sur la vie professionnelle des participantes. D'autres 
"...que les mères

d'enfants présentant

des besoins spéciaux

ont plus de difficulté à

concilier leur vie

familiale et leur vie

professionnelle... on

ne semble pas calculer

les couts, quand ce

n'est que la mère qui

les assume." études ont démontré que les mères d'enfants présentant des besoins spéciaux ont plus de difficulté à concilier leur vie familiale et leur vie professionnelle (Descarries et Corbeil 1998). Parmi les raisons invoquées, on note le manque de souplesse et l'absence des ressources requises par ces familles (Greenspan 1998). Les participantes soulignent les conflits additionnels qu'elles doivent gérer pour répondre aux crises en milieu scolaire pendant leur journée de travail. À moins de travailler dans un milieu où l'on comprend la nature et l'impact duTHADA, la mère doit constamment s'excuser, expliquer et négocier. Il n'est pas surprenant que plusieurs participantes «choisissent» de faire des sacrifices sur le plan de leur carrière, plutôt que de risquer l'épuisement qui est le sort de quelques-unes des participantes. Cependant, comme l'a rapporté Traustadottir (1991), on ne semble pas calculer les coûts, quand ce n'est que la mère qui les assume.

La présente étude offre plusieurs pistes d'intervention aux travailleuses et travailleurs sociaux desservant les mères d'enfants atteints du THADA. Cette incapacité est la principale raison évoquée pour consulter les services de santé mentale pour enfants (Barkley 1995) et le service social est impliqué auprès de certains enfants, adoptés ou en famille d'accueil, chez qui le THADA se manifeste proportionnellement plus souvent (20 à $30 \%)$ que dans la population générale (Simmel et al. 2000). Certaines études ont trouvé que les familles qui vivent avec cette incapacité connaissent plus de conflits et de divorces (Boyce et al. 1995). De plus, la plupart des familles qui ont recours aux services sociaux ont d'autres problèmes concrets qui s'ajoutent au THADA et qui compliquent la situation.

Afin d'éviter l'épuisement chez les mères et d'empêcher la détérioration familiale, une approche préventive, plutôt que réactive comme celle qui domine maintenant, s'impose. Compte tenu du manque de compréhension et de services pour les familles, les mères sont obligées de «foncer» ou d'attendre qu'une crise attire l'attention des services sociaux ou de santé. Pour changer cette situation et sensibiliser la direction des agences à l'importance de l'intervention précoce dans ces cas, les intervenantes et intervenants doivent se renseigner au sujet duTHADA et de son impact 


\section{"Il faudrait établir des partenariats avec les groupes d'entraide qui défendent les droits de ces familles..."}

sur la famille. Des connaissances précises sont essentielles, non seulement pour contrer les fausses représentations de cette incapacité par les médias, mais aussi pour éviter que les parents se sentent blâmés par des travailleuses ou travailleurs sociaux, tel que rapporté dans l'étude de Viola (1997).

Dans leurs interventions, il est important pour les travailleuses et travailleurs sociaux d'aider les mères non seulement à accéder aux ressources ou à résoudre les crises, mais aussi de réfléchir à la charge globale du travail qu'elles accomplissent. De même, on peut discuter d'options face à l'emploi, afin de favoriser des décisions satisfaisantes. Lorsqu'on fait des suggestions sur le plan de changements à effectuer à la maison, il faut s'assurer que ces nouvelles responsabilités ne tombent pas uniquement sur ces mères déjà surchargées. D'ailleurs, on peut essayer de promouvoir un partage plus équitable des tâches entre les parents, en sachant qu'une plus grande implication paternelle est bénéfique pour toute famille dont un enfant a des besoins spéciaux, en plus d'augmenter la satisfaction et l'harmonie conjugale (Willoughby et Glidden 1995). De même, il est important d'évaluer avec les mères leur réseau de soutien. Si celui-ci n'offre pas beaucoup de soutien, on peut aider au développement d'autres liens.

Si ces interventions peuvent aider les mères à mieux vivre leur situation difficile, il importe de participer aux efforts en vue de faire connaitre les besoins de ces mères. Il faudrait établir des partenariats avec les groupes d'entraide qui défendent les droits de ces familles (Breton 1999) en plus de participer à des efforts de réactivation de tels groupes pour les Franco-Ontariennes. Si ces dernières peuvent faire des alliances stratégiques avec les groupes anglophones comme CHADD pour certaines causes communes telles le répit, il est important qu'elles se retrouvent entre elles pour contrer leur isolement et pour revendiquer les services en français. Cependant, il n'est pas réaliste d'ajouter ce travail bénévole au fardeau qu'assument déjà ces femmes. Elles ont besoin de l'aide des intervenantes et intervenants dans la recherche de subventions pour assurer une permanence à de tels groupes.

Enfin, plusieurs participantes ont mentionné le besoin de sensibiliser la communauté, le personnel scolaire et les milieux 
d'emploi à leur réalité. Selon ces mères, les campagnes médiatiques et des sessions d'information pour le public pourraient détruire certains mythes entourant le THADA et la prise de médicaments. De plus, elles feraient réaliser la somme de travail et de temps exigée par les soins, afin d'éviter que les mères ne soient pénalisées au travail. En plus de participer à l'organisation de telles sessions, les intervenantes et intervenants sociaux pourraient sensibiliser leurs propres milieux d'emploi aux besoins des familles.

"L'administration et les syndicats ont besoin d'information sur les changements requis, tant dans le climat au travail que dans les politiques familiales des milieux d'emploi..."
L'administration et les syndicats ont besoin d'information sur les changements requis, tant dans le climat au travail que dans les politiques familiales des milieux d'emploi, avant que les mères d'enfants ayant le THADA puissent se sentir à l'aise en combinant le travail rémunéré et familial.

Cette étude permet de rendre visible le vécu et les besoins d'un groupe de mères peu connu à ce jour. Si certains résultats ne s'appliquent qu'à celles vivant avec le THADA, d'autres reflètent probablement la réalité de mères dont les enfants ont d'autres incapacités. Nous espérons que suivront d'autres recherches traitant des besoins de telles familles, lesquelles recherches contribueront à leur tour à trouver des façons de mieux les desservir.

\section{Bibliographie}

ANTHONY et Gene FISHER (2001). «Lessons learned», The ADDovcate, vol. 17, 1-3.

BAKER, David et Kevin, McCAL (2005). «Parenting stress in parents of children with attentiondeficit hyperactivity disorder and parents of children with learning disabilities», Journal of child and family studies, vol. 4, no 1,57-68.

BALDWIN, Kevin, Ronald BROWN et Michael MILAN (1995). «Predictors of stress in caregivers of attention deficit hyperactivity disordered children", American Journal of Family Therapy, vol. 23, no 2, 149-160.

BARKLEY, Russell (1995). Taking charge of ADHD, New York, Guilford Press.

BARKLEY, Russell (1997). ADHD and the nature of self control, New York, Guilford Press.

BARNETT, Rosalind, Robert BRENNAN et Nancy MARSHALL (1994). "Gender and the relationship between parent role quality and psychological distress: A study of men and women in dual-earning couples", Journal of Family Issues, vol.15, no 2, 229-252.

BARUCH, Grace, Rosalind BARNETT (1986). "Role quality, multiple role involvement, and psychological well-being in midlife women", Journal of personality and social psychology, vol. 51, no 3, 578-585. 
BERNIER, James, Deborah, SIEGEL (1994). «Attention deficit hyperactivity disorder: A family and ecological systems perspective», Families in Society: The Journal of Contemporary Human Services, vol. 75, 142-150.

BIEDERMAN, Joseph et al. (1995). «Family environment risk factors for attention-deficit hyperactivity disorder», Archives of General Psychiatry, vol. 52, 464-470.

BOYCE, Glenna, Brent, MILLER, Karl WHITE et Michael, GODFREY (1995). «Single parenting in families of children with disabilities", Marriage \& family Review, vol. 20, nos 3-4, 389-409.

BRETON, Margot (1998). «Le service social de groupe dans une approche de partenariat», Revue canadienne de service social, vol. 15, no 2, 193-205.

BRISTOL, Marie, James, GALLAGHER et Eric, SCOPLER (1988). «Mothers and fathers of young developmentally disabled and nondisabled boys:Adaptation and spousal support», Developmental, vol. 24, no 3, 441-451.

BULLOCK,Anne, Roxana, Ng, Gillian, Walker et Jacob Muller (1990). «Community care: Ideology and lived experience», dans Ng, R. Walker, G. et J. Muller, J. éd., Community organisation and the Canadian state, Toronto, Gramond, 65-82.

CANCIAN, Francesca et Stacey, OLIKER (2000). Caring and gender, New York, Atlanta.

DESCARRIES, Francine et Christine, CORBEIL (1998). La valse d'hésitation des mères en emploi: d'hier à aujourd'hui, Quatrième symposium québécois sur la famille, Sillery.

DEVAULT, Marjorie (1999). "Comfort and struggle. Emotion work in family life», the Annals of the American Academy of Political and Social Science, vol. 56, 52-63.

FEWELL Rebecca, (1990). «Parenting moderately handicapped persons», dans M. Seligman, éd., The family with a handicapped child, Boston, Allyn and Bacon, 203-236.

FLOYD, Franklin et Eric GALLAGHER (1997). «Parental stress, care demands, and use of support services for school-age children with disabilities and behavior problems», Family Relations, vol. 46, no 4, 359-371.

GAGNON, André (2001). Démystifier les maladies mentales, les troubles de l'enfance et de l'adolescence, Boucherville, Gaëtan Morin éditeur.

GERSON, Kathleen (1998). «Gender and the future of the family: Implication for the post industrial workplaces», dans D et P.Vanney, D et P, Dubeck, éd., Challenges for work and family in the twenty first century, New York, Aldine de Gruyter.

GREENSPAN, Miriam. (1998). «Exceptional mothering in a normal world», dans Garcia Coll., C. Surrey, J. et K. Weingarten, éd. Mothering Against the Odds, New York, Guilford, 37-60.

HOME, Alice (1997). «Learning the hard way: Role strain, stress, role demands, and support in multiple-role women students», Journal of Social Work Education, vol. 33, no 2.

HOME, Alice et Stephanie PEARCE, Stephanie (2002). «Mothering children with ADHD: What we've learned so far», The Canadian, 12-14.

HUBERMAN, Michael et Matthew MILLES (1991). Analyse de données qualitatives. Recueil de nouvelles méthodes, traduction de l'anglais par Catherine De Backer et Vivian Lamongie, Bruxelles, De Boeck, Wesmael.

INSTITUTVANIER DE LA FAMILLE (1998). It's the kitchen to the boardroom table, Ottawa, Institut Vanier.

JACOBS, Edward (1998). Fathering the ADHD child, Northvale, Aronson. 
JENKINS, David (1997). «Women, work and care giving. How do these roles affect women's wellbeing?", Journal of women and aging, vol. 9, no 3, 27-45.

L'ÉCUYER, René (1985). «L'analyse de contenu. Notions et étapes», dans Jean-Pierre Deslauriers,

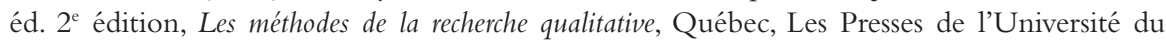
Québec, 65-84.

LEE, D. (1998). Having it all, having enough, New York, Amacom.

MACDONALD, T, POETNER et J., PIERPONT (1999). «Predicting caregiver stress:An ecological perspective", American Journal of Orthopsychiatry, vol. 9, no 1, 100-109.

MARCENKO, Maureen et Judith, MEYERS(1991). «Mothers of children with developmental disabilities: Who shares the burden?», Family Relations, vol. 40, 186-190.

MARDIROS, Marilyn. (1985). «Role alterations of female parents having children with disabilities», Canada's Mental Health, vol. 33, no 4, 24-26.

PATTON, Michael (1994). Qualitative evaluation and research methods, Newbury Park, Sage.

ROCHER Institute (2000). Beyond the limits: Mothers caring for children with disabilities, North York, Ont., L'institut Rocher Institute.

SELIGMAN, Milton et Rosalyn, DARLING (1997). Ordinary families, special children. $2^{\text {nd }}$, New York, Guilford.

SHEARN, Julia et Stuart, TODD (2000). «Maternal employment and family responsibilities: The perspective of mothers of children with intellectual disabilities», Journal of Applied Research in Intellectual Disabilities, vol. 13, 109-131.

SIMMEL, Edward, Daniel, BROOKS et S., HINDSHAW (2001). «Externalizing symptomatology among adoptive youth: Prevalence and preadoption risk factors», Journal of Emotional and Behavioral Disorders, vol. 5, no 4, 223-229.

SLOPER, Patricia (1999). "Models of service support for parents of disabled children. What we know? What we need to know?», Child Care, Health \& Development, vol. 25, no 2, 85-99.

SNYDER, Marlene (2000). "AD/HD and co-existing disorders ACHADD fact sheet», The ADDvocate, 15.

TÉTREAULT, Sylvie et Linda BLANCHETTE (1991). «Réflexion sur la condition de la mère de l'enfant handicapé: une intervention féministe à développer», Service social, vol. 40, no 2,117143.

TRAUSTADOTTIR, Rannveig (1991). «Mothers who care: Gender, disability, and family life», Journal of Family Issues, vol. 1, no 2, 211-228.

VIOLA, Sylvie (1997). Concerns and perceptions of parents of children and adolescents with Attentiondeficit-hyperactivity-disorder, Unpublished doctoral thesis, Indiana State University.

WILLOUGHBY,Jennifer et Loraine, MARSTER-GLIDDEN (1995). «Fathers helping out: Shared child care and marital satisfaction of parents of children with disabilities", American Journal on Mental Retardation, vol. 99, no 4, 399-406.

\section{Notes}

1. Nous remercions le Conseil de recherche en sciences humaines du Canada, qui a subventionné cette étude. 